\title{
CALCULATION OF DENSITY FOR THE MULTIVARIATE POISSON DISTRIBUTION
}

\author{
By KazUTOMO KaWAMURA
}

\section{§1. Summary.}

On the bivariate case, each Poisson density has five different recurrence relations, in the followin lines, we denote them as r.r's. and conversely, if we need to calculate the density, we can omit one of the two main relations. On the trivariate case, there are 13 different $r . r$ 's. Let us put the $r . r$ 's in the concrete, on the bivariate case.

It is known that a density function of Poisson distribution $p(k, l)(k, l=0$, $1,2, \cdots)$ satisfies two main recurrence relations:

$$
\begin{aligned}
& k p(k, l)=\lambda_{10} p(k, 1-l)+\lambda_{11} p(k-1, l-1), \\
& l p(k, l)=\lambda_{01} p(k-1, l)+\lambda_{11} p(k-1, l-1),
\end{aligned}
$$

Conversely, if we need to calculate the density using the $r . r$ 's., not using the direct calculation, one of the relations would be meaning less except in the usage of its reduced relations:

$$
\begin{gathered}
k p(k, 0)=\lambda_{10} p(k-1,0), \quad l p(0, l)=\lambda_{01} p(0, l-1) \\
\text { and } p(0,0)=\exp \left(-\lambda_{10}-\lambda_{01}-\lambda_{11}\right) .
\end{gathered}
$$

On the trivariate case, we have three main recurrence relations $(r . r$ 's.):

$$
\begin{aligned}
k p(k, l, m)= & \lambda_{100} p(k-1, l, m)+\lambda_{110} p(k-1, l-1, m) \\
& +\lambda_{101} p(k-1, l, m-1)+\lambda_{111} p(k-1, l-1, m-1), \\
l p(k, l, m)= & \lambda_{010} p(k, l-1, m)+\lambda_{110} p(k-1, l-1, m) \\
& +\lambda_{011} p(k, l-1, m-1)+\lambda_{111} p(k-1, l-1, m-1), \\
m p(k, l, m)= & \lambda_{001} p(k, l, m-1)+\lambda_{101} p(k-1, l, m-1) \\
& +\lambda_{011} p(k, l-1, m-1)+\lambda_{111} p(k-1, l-1, m-1) .
\end{aligned}
$$

To calculate the density $p(k, l, m)$ from the $r . r$ 's. we need one of the rela-

Received December 12, 1986 
tion and their reduced relations.

In this paper we will express the r.r's. for the multivarivate case and it will be shown that if we need to calculate the density we use only one of the $n$ main relations and their reduced relations. Because they include the other main relations as shown in the last section of this paper.

\section{$\S 2$. Notations and Definitions.}

$p(k ; \lambda)$ : univariate Poisson density with parameter $\lambda$

$\boldsymbol{i}=\left(i_{1}, i_{2}, \cdots, \imath_{n}\right) \imath_{\jmath}=0$ or $1(j=1,2, \cdots, n)$

$\boldsymbol{k}=\left(k_{1}, k_{2}, \cdots, k_{n}\right) k_{j} \geqq 0$ nonnegative integer for $\jmath=1,2, \cdots, n$

$\boldsymbol{O}=(0,0, \cdots, 0)$

$\boldsymbol{E}_{0}=\{0,1\}^{n}, \boldsymbol{E}=\{0,1\}^{n}-0$

$p(\boldsymbol{k}): n$-variate Poisson density

$\alpha_{i}, \beta_{i}$ : nonnegative integers

$\alpha=\left\{\alpha_{\imath}, i \in E\right\}$ vector with nonnegative components $\alpha_{i}$ for some ordering of $i$, say, the linary scale.

$[C(\boldsymbol{k})]$ : a restriction of $\alpha$ depending only on $k$ a set of $\alpha$

$\boldsymbol{p}=\left\{p_{\boldsymbol{i}} ; \boldsymbol{i} \in E_{0}\right\}$ vector with $2^{n}$ components of probabilities such that $\sum_{i \in E 0} p_{i}=1\left(\begin{array}{l}* \\ *\end{array}\right)$

$\lambda=\left\{\lambda_{\imath} ; \boldsymbol{i} \in E\right\}$ vector with $2^{n}-1$ nonnegative parameters.

$\# \boldsymbol{k}$ : the number of positive components of a vector $\boldsymbol{k}$

-calculable, $p_{0}$-calculable-

$p(\boldsymbol{k})$ is calculable on $S$ : If we can calculate all the values $p(\boldsymbol{k})$ for $\boldsymbol{k} \in S$ from some prescribed relations then we call $p(\boldsymbol{k})$ to be "calculable on $S$ " or simply "calculable". In the same way, if we can calculate all the values $p(\boldsymbol{k})$ for $\boldsymbol{k} \in S-(0,0)$ from some prescribed relations except the boundary condition indicating the value of origin $p_{0}$, we call $p(\boldsymbol{k})$ for $\boldsymbol{k} \in S-(0,0)$ to be " $p_{0}$-calculable on $S$ " or simply " $p_{0}$-calculable".

$[C(\boldsymbol{k})]$ : a set of vectors $\alpha=\left\{\alpha_{\imath}, i \in E\right\}$ with a restriction listed as below, and we will shorten it as $[C]$

$$
[C]=[C(\boldsymbol{k})]=\left\{\alpha ; \sum_{\boldsymbol{\imath} \neq 0} i \alpha_{\imath}=\boldsymbol{k}\right\}
$$

where $\boldsymbol{i} \neq 0$ means $\boldsymbol{i} \in E$ and $0 \neq \boldsymbol{i} \leqq \boldsymbol{k}$ and $\sum_{[C]} u_{\alpha}$ means the sum of $u_{\alpha}$ where $\alpha$ varies on the set $[C]$.

$n$-variate Poisson density:

$$
p(\boldsymbol{k})=\sum_{[C]} \prod_{i \in E} p\left(\alpha_{\imath} ; \lambda_{\imath}\right),
$$

we denote this distribution as $P(\lambda)$. If $X$ is a $n$-variate random vector with the distribution $P(\lambda)$ then we can express $X$ as 


$$
X=\sum_{i \in E} i X_{\imath}
$$

where $X_{i}$ is an univariate Poisson distribution $P\left(\lambda_{2}\right)$ and $X_{i}$ are mutually independent for $\boldsymbol{i} \in E$, see Kawamura [4].

$\boldsymbol{k} \geqq \boldsymbol{i}:$ A relation of two vectors of both sides, which means any two corresponding components $k_{\jmath}$ and $i$, satisfy $\boldsymbol{k}_{\jmath} \geqq \boldsymbol{i}_{\text {, }}$. We use the relation as $0 \leqq \boldsymbol{i} \leqq \boldsymbol{k}$.

\section{§ 3. Introduction.}

On the bivariate case, the density function of $P(\lambda)$ is expressed as

$$
p(\boldsymbol{k}, \boldsymbol{l})=\sum_{\delta=0}^{k \wedge l} P\left(\boldsymbol{k}-\delta ; \lambda_{10}\right) P\left(\boldsymbol{l}-\delta ; \lambda_{01}\right) P\left(\delta ; \lambda_{11}\right)
$$

for any nonnegative integers $k, l$ and we used $\boldsymbol{k} \triangle \boldsymbol{l}=\min (\boldsymbol{k}, \boldsymbol{l})$, see Kawamura [2] and Polak [7].

The density function $p(\boldsymbol{k}, \boldsymbol{l})$ : a nonnegative function defined on the lattice points of the first quadrant including two axes, satisfies following recurrence relations, see Johnson and Kotz [1].

$$
\begin{aligned}
& \boldsymbol{k} p(\boldsymbol{k}, \boldsymbol{l})=\lambda_{10} p(\boldsymbol{k}-1, \boldsymbol{l})+\lambda_{11} p(\boldsymbol{k}-1, \boldsymbol{l}-1) \\
& \boldsymbol{l} p(\boldsymbol{k}, \boldsymbol{l})=\lambda_{01} p(\boldsymbol{k}, \boldsymbol{l}-1)+\lambda_{11} p(\boldsymbol{k}-1, \boldsymbol{l}-1)
\end{aligned}
$$

It is better to express the relations as followings, because of they don't include the reduced forms of at least one of $k$ and $l$ equals zero.

$$
\begin{array}{ll}
\boldsymbol{k}=\boldsymbol{l}=0 & p(0,0)=\exp \left(-\lambda_{10}-\lambda_{01}-\lambda_{11}\right) \\
\boldsymbol{k} \geqq 1, \boldsymbol{l}=0 & \boldsymbol{k} p(\boldsymbol{k}, 0)=\lambda_{10} p(\boldsymbol{k}-1,0) \\
\boldsymbol{k}=0, \boldsymbol{l} \geqq 1 & \boldsymbol{l} p(0, \boldsymbol{l})=\lambda_{01} p(0, \boldsymbol{l}-1) \\
\boldsymbol{k}, \boldsymbol{l} \geqq 1 & \boldsymbol{k} p(\boldsymbol{k}, \boldsymbol{l})=\lambda_{10} p(\boldsymbol{k}-1, \boldsymbol{l})+\lambda_{11} p(\boldsymbol{k}-1, \boldsymbol{l}-1) \\
\boldsymbol{k}, \boldsymbol{l} \geqq 1 & \boldsymbol{l} p(k, \boldsymbol{l})=\lambda_{01} p(\boldsymbol{k}, \boldsymbol{l}-1)+\lambda_{11} p(\boldsymbol{k}-1, \boldsymbol{l}-1) .
\end{array}
$$

The first equality (1) is not a relation but explains the density. If we need to calculate the density $p(\boldsymbol{k}, \boldsymbol{l})(\boldsymbol{k}, l \geqq 0)$, we can calculate $p(0,0)$ by $(1)$ and $p(\boldsymbol{k}, 0)(\boldsymbol{k} \geqq 1)$ are calculable from (2) and the induction for $k$ and also $p(0, l)$ $(\boldsymbol{l} \geqq 1)$ are calculable from the same argument. For $p(\boldsymbol{k}, \boldsymbol{l})(\boldsymbol{k}, \boldsymbol{l} \geqq 1)$ we need one of the main relations (4) and (5). That is, we don't need one of the other relation to prove the calculability of the density $p(\boldsymbol{k}, \boldsymbol{l})(\boldsymbol{k}, \boldsymbol{l} \geqq 0)$, see Kawamura [5].

In the case of trivariate Poisson distribution $P(\lambda)$. The density is written as

$$
p(\boldsymbol{k})=p\left(\boldsymbol{k}_{1}, \boldsymbol{k}_{2}, \boldsymbol{k}_{3}\right)=\sum_{\lceil C]} \prod_{i \neq 0} p\left(\alpha_{\imath}, \lambda_{\boldsymbol{\imath}}\right) \quad(\boldsymbol{k} \geqq 0)
$$


for any pair of nonnegative integers $\boldsymbol{k}_{1}, \boldsymbol{k}_{2}, \boldsymbol{k}_{3}$, see for Kawamura [3] and Liu [6], where $\boldsymbol{k}=\left(k_{1}, k_{2}, k_{3}\right)$ and $\boldsymbol{k} \geqq 0$ means every component $k_{j}(j=1,2,3)$ is nonnegative. And for given $\boldsymbol{k} \geqq 0$, [C] means a set of pair $\alpha=\left\{\alpha_{\boldsymbol{\imath}} ; \boldsymbol{i} \in E\right\}$ having a restriction listed as follows

$$
[C]=\left\{\alpha ; \sum_{i \neq 0} i \alpha_{i}=k\right\}
$$

and $\sum_{[C]} u_{\alpha}$ means the sum of $u_{\alpha}$ where $\alpha$ varies on the set $[C]$. Another expression for $[C]$ :

$$
\begin{aligned}
\sum_{\boldsymbol{i} \neq 0} \boldsymbol{i} \alpha_{\boldsymbol{i}}=k & \Leftrightarrow \sum_{i_{1}=1} \boldsymbol{i}_{1} \alpha_{i}=\boldsymbol{k}_{1}, \quad \sum_{\boldsymbol{i}_{2}=1} \boldsymbol{i}_{2} \alpha_{\boldsymbol{i}}=\boldsymbol{k}_{2}, \quad \sum_{\boldsymbol{i}_{3}=1} \boldsymbol{i}_{3} \alpha_{\boldsymbol{i}}=\boldsymbol{k}_{3} \\
& \Leftrightarrow \sum_{\boldsymbol{i}_{j}=1} \alpha_{i}=\boldsymbol{k}_{3} \quad(j=1,2,3) .
\end{aligned}
$$

THEOREM 3-1. For given trivariate Poisson density $p(\boldsymbol{k})(\boldsymbol{k} \geqq 0)$, we have an expression of the recurrence relations

$$
\begin{aligned}
& \boldsymbol{k}=0 ; \quad p(0)=\prod_{\boldsymbol{i} \in E} p\left(0 ; \lambda_{\boldsymbol{\imath}}\right)=\exp \left(-\sum_{\boldsymbol{i} \in E} \lambda_{\boldsymbol{\imath}}\right) \\
& \boldsymbol{k} \neq 0 ; \quad \boldsymbol{k}_{\jmath} p(\boldsymbol{k})=\sum_{\boldsymbol{i}_{j}=1, \boldsymbol{i} \leq \boldsymbol{k}} \lambda_{\boldsymbol{i}} p(\boldsymbol{k}-\boldsymbol{i})
\end{aligned}
$$

for $j$ such that $\boldsymbol{k} j \geqq 1(j=1,2, \cdots, n)$.

We can combine the last equation as

$$
\boldsymbol{k} p(\boldsymbol{k})=\sum_{\boldsymbol{\imath} \in E, i \leq k} i \lambda_{\imath} p(k-i)
$$

We have a concrete expression of the recurrence relations as given in the next corollary.

COROLlary 3-1. For given trivariate Porsson density $p(\boldsymbol{k})(\boldsymbol{k} \geqq 0)$, we have a concrete expression of the r.r's:

$$
\begin{array}{ll} 
& p(0,0,0)=\exp \left(-\lambda_{100}-\lambda_{010}-\lambda_{001}-\lambda_{110}-\lambda_{101}-\lambda_{011}-\lambda_{111}\right) \\
\boldsymbol{k}_{1} \geqq 1 & \boldsymbol{k}_{1} p\left(k_{1}, 0,0\right)=\lambda_{100} p\left(k_{1}-1,0,0\right) \\
\boldsymbol{k}_{2} \geqq 1 & \boldsymbol{k}_{2} p\left(0, \boldsymbol{k}_{2}, 0\right)=\lambda_{010} p\left(0, \boldsymbol{k}_{2}-1,0\right) \\
\boldsymbol{k}_{3} \geqq 1 & \boldsymbol{k}_{3} p\left(0,0, \boldsymbol{k}_{3}\right)=\lambda_{001} p\left(0,0, \boldsymbol{k}_{3}-1\right) \\
\boldsymbol{k}_{2}, \boldsymbol{k}_{3} \geqq 1 & \boldsymbol{k}_{2} p\left(0, \boldsymbol{k}_{2}, \boldsymbol{k}_{3}\right)=\lambda_{010} p\left(0, \boldsymbol{k}_{2}-1, \boldsymbol{k}_{3}\right)+\lambda_{011} p\left(0, \boldsymbol{k}_{2}-1, \boldsymbol{k}_{3}-1\right) \\
& \boldsymbol{k}_{3} p\left(0, \boldsymbol{k}_{2}, \boldsymbol{k}_{3}\right)=\lambda_{001} p\left(0, \boldsymbol{k}_{2}, \boldsymbol{k}_{3}-1\right)+\lambda_{011} p\left(0, \boldsymbol{k}_{2}-1, \boldsymbol{k}_{3}-1\right) \\
\boldsymbol{k}_{3}, \boldsymbol{k}_{1} \geqq 1 & \boldsymbol{k}_{3} p\left(\boldsymbol{k}_{1}, 0, \boldsymbol{k}_{3}\right)=\lambda_{001} p\left(\boldsymbol{k}_{1}, 0, \boldsymbol{k}_{3}-1\right)+\lambda_{101} p\left(\boldsymbol{k}_{1}-1,0, \boldsymbol{k}_{3}-1\right)
\end{array}
$$




$$
\begin{aligned}
\boldsymbol{k}_{1} p\left(\boldsymbol{k}_{1}, 0, \boldsymbol{k}_{3}\right)=\lambda_{100} p\left(\boldsymbol{k}_{1}-1,0, \boldsymbol{k}_{3}\right)+\lambda_{101} p\left(\boldsymbol{k}_{1}-1,0, \boldsymbol{k}_{3}-1\right) \\
\boldsymbol{k}_{1}, \boldsymbol{k}_{2} \geqq 1 \quad \boldsymbol{k}_{1} p\left(\boldsymbol{k}_{1}, k_{2}, 0\right)=\lambda_{100} p\left(\boldsymbol{k}_{1}-1, \boldsymbol{k}_{2}, 0\right)+\lambda_{110} p\left(\boldsymbol{k}_{1}-1, \boldsymbol{k}_{2}-1,0\right) \\
\boldsymbol{k}_{2} p\left(\boldsymbol{k}_{1}, \boldsymbol{k}_{2}, 0\right)=\lambda_{010} p\left(\boldsymbol{k}_{1}, \boldsymbol{k}_{2}-1,0\right)+\lambda_{110} p\left(\boldsymbol{k}_{1}-1, \boldsymbol{k}_{2}-1,0\right) \\
\boldsymbol{k}_{1}, \boldsymbol{k}_{2}, \boldsymbol{k}_{3} \geqq 1 \quad \boldsymbol{k}_{1} p\left(\boldsymbol{k}_{1}, \boldsymbol{k}_{2}, \boldsymbol{k}_{3}\right)=\lambda_{100} p\left(\boldsymbol{k}_{1}-1, \boldsymbol{k}_{2}, \boldsymbol{k}_{3}\right)+\lambda_{110} p\left(\boldsymbol{k}_{1}-1, \boldsymbol{k}_{2}-1, \boldsymbol{k}_{3}\right) \\
+\lambda_{101} p\left(\boldsymbol{k}_{1}-1, \boldsymbol{k}_{2}, \boldsymbol{k}_{3}-1\right)+\lambda_{111} p\left(\boldsymbol{k}_{1}-1, \boldsymbol{k}_{2}-1, \boldsymbol{k}_{3}-1\right) \\
\boldsymbol{k}_{2} p\left(\boldsymbol{k}_{1}, \boldsymbol{k}_{2}, \boldsymbol{k}_{3}\right)=\lambda_{010} p\left(\boldsymbol{k}_{1}, \boldsymbol{k}_{2}-1, \boldsymbol{k}_{3}\right)+\lambda_{110} p\left(\boldsymbol{k}_{1}-1, \boldsymbol{k}_{2}-1, \boldsymbol{k}_{3}\right) \\
+\lambda_{011} p\left(\boldsymbol{k}_{1}, \boldsymbol{k}_{2}-1, \boldsymbol{k}_{3}-1\right)+\lambda_{111} p\left(\boldsymbol{k}_{1}-1, \boldsymbol{k}_{2}-1, \boldsymbol{k}_{3}-1\right) \\
\boldsymbol{k}_{3} p\left(\boldsymbol{k}_{1}, \boldsymbol{k}_{2}, \boldsymbol{k}_{3}\right)=\lambda_{001} p\left(\boldsymbol{k}_{1}, \boldsymbol{k}_{2}, \boldsymbol{k}_{3}-1\right)+\lambda_{101} p\left(\boldsymbol{k}_{1}-1, \boldsymbol{k}_{2}, \boldsymbol{k}_{3}-1\right) \\
+\lambda_{011} p\left(\boldsymbol{k}_{1}, \boldsymbol{k}_{2}-1, \boldsymbol{k}_{3}-1\right)+\lambda_{111} p\left(\boldsymbol{k}_{1}-1, \boldsymbol{k}_{2}-1, \boldsymbol{k}_{3}-1\right) .
\end{aligned}
$$

Conversely, if we calculate the density function $p(\boldsymbol{k})(\boldsymbol{k} \geqq 0)$ from the relations, we need only one of 3 main relations $(11) \sim(13)$ and their reduced relations. If we search deligently we have 13 relations and we need only specified 8 from the 13 relations to calculate the density conversely.

THEOREM 3-2 (8 relations). If nonnegative function $q(\boldsymbol{k})$ defined on $\boldsymbol{k} \geqq 0$ satisfy (1), (2), (3), (4), (5) or (6), (7) or (8), (9) or (10) and (11) or (12) or (13) then $q(\boldsymbol{k})$ consists with the density of the Poisson distribution $p(\boldsymbol{k})(\boldsymbol{k} \geqq 0)$.

This theorem means the minimal r.r's. are (1) (4) and one of (5) and (6), and one of (7) and (8), and one of (9) and (10), and one of (11), (12) and (13) to get the calculablity.

COROLLARY 3-2 (7 relations). If a density functions $q(\boldsymbol{k})$ on $k \geqq 0$ satisfy (2) $\sim(4)$, (5) or (6), (7) or (8), (9) or (10) and (11) or (12) or (13) then $q(\boldsymbol{k})$ consists with one of the density $p(\boldsymbol{k})$ on $\boldsymbol{k} \geqq 0$.

\section{§4. Multivariate Poisson distribution and its recurrence relations.}

We assume $X_{0}$ to be $n$-variate bivariate distribution $B(N, p), X_{0}$ is explained as the sum of $N$ independent distributions $B(1, p)$. We can derive $n$-variate Poisson distribution $P(\lambda)$ by the limiting distribution of $X_{0}$ with the restriction $N p_{i} \rightarrow \lambda_{i}$ as $N \rightarrow \infty$ for every $i \in E$. Denote $X$ as the $n$ variate Poisson distribution $P(\lambda)$ then we can represent the density

$$
p(X=\boldsymbol{k})=\sum_{[C]} \prod_{i \neq 0} p\left(\alpha_{\imath}, \lambda_{\boldsymbol{\imath}}\right)
$$

where $p\left(\alpha_{\boldsymbol{\imath}}, \lambda_{\boldsymbol{\imath}}\right)(\boldsymbol{i} \in E)$ are usual univariate Poisson density 


$$
p\left(\alpha_{i}, \lambda_{1}\right)=\left(\lambda_{1} / \alpha_{i} !\right) \exp \left(-\lambda_{i}\right) \quad(i \in E)
$$

and $[C]$ should be generalized to $n$-variate $\alpha$ set

$$
[C]=\left\{\alpha ; \sum_{i \neq 0} i \alpha_{i}=k\right\},
$$

see Kawamura [4]. Then we have a recurrence relation of $P(\lambda)$

$$
\boldsymbol{k} p(\boldsymbol{k})=\sum_{0 \leqq \imath \leq k} i \lambda_{i} p(\boldsymbol{k}-\boldsymbol{i})
$$

and if $\boldsymbol{k}=0$ we have $p(0)=\prod_{i \in E} p\left(0, \lambda_{\boldsymbol{\imath}}\right)=\exp \left(-\sum_{i \in E} \lambda_{\boldsymbol{i}}\right)$ or we can express $(*)$ as

$$
\boldsymbol{k}_{j} p(\boldsymbol{k})=\sum_{\boldsymbol{i}_{j}=1, i \leq k} \lambda_{i} p(\boldsymbol{k}-\boldsymbol{i})
$$

where $\boldsymbol{k}_{j} \geqq 1$ for some $j=1,2, \cdots, n$. We can summarize these relations as following.

Theorem 4-1. The density $p(\boldsymbol{k})$ of n-variate Porsson distribution $P(\lambda)$ satisfies the recurrence relations

and

$$
p(0)=\exp \left(-\sum_{\imath \in E} \lambda_{\imath}\right)
$$

$$
\boldsymbol{k} p(\boldsymbol{k})=\sum_{0 \leqq \imath \leqq k} i \lambda_{i} p(\boldsymbol{k}-i)
$$

Proof. If $\boldsymbol{k}_{j} \geqq 1$ then

$$
\begin{aligned}
& \boldsymbol{k}_{j}=\left[\sum_{\boldsymbol{i} \in E} i \alpha_{i}\right]_{j}=\sum_{i \in E} i_{j} \alpha_{i}=\sum_{\boldsymbol{t}_{j}=1} \alpha_{\boldsymbol{i}}, \\
& \boldsymbol{k}, p(\boldsymbol{k})=\boldsymbol{k}, \sum_{[C]} \prod_{\boldsymbol{i} \neq 0} p\left(\alpha_{\boldsymbol{\imath}}, \lambda_{\boldsymbol{i}}\right) \\
& =\sum_{[C]}\left(\sum_{i_{j}=1} \alpha_{\imath}\right) \prod_{i \neq 0} p\left(\alpha_{i}, \lambda_{i}\right) \\
& =\sum_{[C]} \sum_{i_{j}=1} \lambda_{2} \prod_{i \neq 0} p\left(\beta_{i}, \lambda_{i}\right) \quad(\jmath=1,2, \cdots, n),
\end{aligned}
$$

where $\beta_{i}=\alpha_{i}-1$ with $i$ satisfying $\boldsymbol{i}_{j}=1$ and otherwise $\beta_{i}=\alpha_{\imath}$.

$$
\begin{aligned}
\boldsymbol{k}_{\jmath} p(\boldsymbol{k}) & =\sum_{\boldsymbol{i}_{j}=1} \lambda_{\boldsymbol{l}} \sum_{[C]} \prod_{\boldsymbol{i} \neq 0} p\left(\beta_{\boldsymbol{i}}, \lambda_{\boldsymbol{\imath}}\right) \\
& =\sum_{\boldsymbol{i}_{j}=1} \lambda_{\boldsymbol{i}} p(\boldsymbol{k}-\boldsymbol{i}) \quad(j=1,2, \cdots, n),
\end{aligned}
$$

where $p\left(\beta_{i}, \lambda_{i}\right)=0$ for $\beta_{i}=-1$. We can summarize these relations as

$$
\boldsymbol{k} p(\boldsymbol{k})=\sum_{0 \leq i \leq k} i \lambda_{i} p(k-i) \quad k \neq 0 .
$$


The recurrence relations just proved involves $n 2^{n-1}$ different relations which are caused on the boundary conditions regarding $k$. And if $k=0$ then we have directly from the density

$$
p(0)=\prod_{i \cong E} p\left(0 ; \lambda_{\boldsymbol{\imath}}\right)=\prod_{i \neq 0} \exp \left(-\lambda_{\boldsymbol{\imath}}\right)=\exp \left(-\sum_{\boldsymbol{\imath} \neq 0} \lambda_{\boldsymbol{\imath}}\right) .
$$

In the case if $\boldsymbol{k} \neq 0$ we could classify $\boldsymbol{k}$ by the number of positive components of the vector as followings.

If $\sharp k=1$, that is, only one of the components $k_{j} \geqq 1$ then we have

$$
\boldsymbol{k}_{\jmath} p(\boldsymbol{k})=\lambda_{\imath} p(\boldsymbol{k}-\boldsymbol{i}) \quad(0 \neq \boldsymbol{i} \leqq \boldsymbol{k})
$$

where $\boldsymbol{i} \leqq \boldsymbol{k}$ and $\boldsymbol{i} \neq 0$ include only one $\boldsymbol{i}$ such that $\boldsymbol{i}_{\jmath}=1$ otherwise $\boldsymbol{i}_{\boldsymbol{k}}=0$ for $k \neq \jmath$. Practically we have for $\sharp \boldsymbol{k}=1$,

$$
\boldsymbol{k}_{j} p\left(0 \cdots 0 \boldsymbol{k}_{j}, \cdots 0\right)=\lambda_{0 \cdots 010 \cdots 0} p\left(0 \cdots 0 \boldsymbol{k}_{j}-10 \cdots 0\right) .
$$

We can vary $j$ from 1 to $n$, so we have $n$ relations for $\sharp k=1$. The number of relations would be $\left(\begin{array}{l}n \\ 1\end{array}\right)$, having one term in the right side.

Also, in the case $\sharp \boldsymbol{k}=2\left(\boldsymbol{k}_{\boldsymbol{\imath}}, \boldsymbol{k}_{j} \geqq 1, \boldsymbol{i}<j\right)$, we have practically

$$
\begin{gathered}
\boldsymbol{k}_{\jmath} p\left(0 \cdots 0 \boldsymbol{k}_{\boldsymbol{i}} 0 \cdots 0 \boldsymbol{k}_{\jmath} 0 \cdots 0\right)=\lambda_{0 \cdots 010 \cdots 0000} p\left(0 \cdots 0 \boldsymbol{k}_{\boldsymbol{i}}-10 \cdots 0 \boldsymbol{k}_{\jmath} 0 \cdots 0\right) . \\
+\lambda_{0 \cdots 010 \cdots 010 \cdots 0} p\left(0 \cdots 0 \boldsymbol{k}_{1}-10 \cdots 0 \boldsymbol{k}_{j}-10 \cdots 0\right) . \\
\boldsymbol{k}_{\jmath} p\left(0 \cdots 0 \boldsymbol{k}_{\boldsymbol{\imath}} 0 \cdots 0 \boldsymbol{k}_{\jmath} 0 \cdots 0\right)=\lambda_{0 \cdots 000 \cdots 010 \cdots 0} p\left(0 \cdots 0 k_{\boldsymbol{\imath}} 0 \cdots 0 \boldsymbol{k}_{j}-10 \cdots 0\right) . \\
+\lambda_{0 \cdots 010 \cdots 010 \cdots 0} p\left(0 \cdots 0 \boldsymbol{k}_{\boldsymbol{i}}-10 \cdots 0 \boldsymbol{k}_{j}-10 \cdots 0\right) .
\end{gathered}
$$

The number of relations would be $\left(\begin{array}{l}n \\ 2\end{array}\right)$, and each has two terms in the right side.

By the induction for $\# k$ we have totally

$$
1\left(\begin{array}{c}
n \\
1
\end{array}\right)+2\left(\begin{array}{c}
n \\
2
\end{array}\right)+\cdots+n\left(\begin{array}{c}
n \\
n
\end{array}\right)=n 2^{n-1}
$$

relations. We can conclude as the theorem.

THEOREM 4-2. The density function $p(\boldsymbol{k})$ on $\boldsymbol{k} \geqq 0$ of $n$-variate Poisson distribution $P(\lambda)$ has totally

$$
1+1\left(\begin{array}{c}
n \\
1
\end{array}\right)+2\left(\begin{array}{c}
n \\
2
\end{array}\right)+\cdots+n\left(\begin{array}{c}
n \\
n
\end{array}\right)=n 2^{n-1}+1
$$

mutually different recurrence relations depending on the boundary condition for $\boldsymbol{k}$. including the density

$$
p(0)=\exp \left(-\sum_{\imath \neq 0} \lambda_{\imath}\right)
$$




\section{$\S 5$. Minimal relations for getting a multivariate Poisson density.}

Let's consider the main theme of calculation of the density. Primarily we will try to calculate directly from the density function but we will risk summing up a lot of accidental error, really we can calculate the density by computer if we make the software being careful not to summarize the error. It is, generally, a very difficalt way to calculate the density and we will be able to improve the situation by these recurrence relations.

To calculate the density $p(\boldsymbol{k})$ of $n$-variate Poisson distribution $P(\lambda)$ we need only prespecified $2^{n}$ relations from the $1+n 2^{n-1}$ relations given in the theorem 4-2. We can express the assertion in the following theorem.

As denoted and defined in the preceding section, we use the notation $\# \boldsymbol{k}$ as the number of positive components of $k=\left(\boldsymbol{k}_{1}, \boldsymbol{k}_{2}, \cdots, \boldsymbol{k}_{n}\right)$, if we can calculate the density $p(\boldsymbol{k})$ for $\boldsymbol{k} \in S$ by some prescribed relations, then we call $p(\boldsymbol{k})$ to be "calculable on $S$ " or simply "calculable".

THEOREM 5-1. The density of n-variate Poisson distribution $p(\boldsymbol{k})$ for $\boldsymbol{k} \geqq 0$ is "calculable" from the prescribed $2^{n}$ relations including the relation

$$
p(0)=\exp \left(-\sum_{i \neq 0} \lambda_{\imath}\right)
$$

In the case of $\# \boldsymbol{k}=0(\boldsymbol{k}=0)$, we have one relation $p(0)=\exp \left(-\sum_{\boldsymbol{i} \neq 0} \lambda_{\boldsymbol{l}}\right)$ we need it to calculate the density.

In the case of $\# \boldsymbol{k}=1$. If $\boldsymbol{k}_{j} \geqq 1$ for fixed $j=1,2, \cdots, n$ we have one relation

$$
\boldsymbol{k}_{\jmath} p\left(0 \cdots 0 \boldsymbol{k}_{j}, \cdots 0\right)=\lambda_{0 \cdots 010 \cdots 0} p\left(0 \cdots 0 \boldsymbol{k}_{j}-10 \cdots 0\right) \text {. }
$$

and we need it to do this. The selection of $j$ may be $\left(\begin{array}{l}n \\ 1\end{array}\right)$ cases then we have totally $\left(\begin{array}{l}n \\ 1\end{array}\right)$ relations and we need all of them to do this.

In the case of $\# \boldsymbol{k}=2$, if $\boldsymbol{k}_{\boldsymbol{\imath}}, \boldsymbol{k}_{j} \geqq 1$ for fixed $\boldsymbol{i}, j ; 1 \leqq \boldsymbol{i}<j \leqq n$ then we have two relations

and

$$
\begin{gathered}
\boldsymbol{k}_{\imath} p\left(0 \cdots 0 \boldsymbol{k}_{\boldsymbol{\imath}} 0 \cdots 0 \boldsymbol{k}_{\jmath} 0 \cdots 0\right)=\lambda_{0 \cdots 010 \cdots 000 \cdots 0} p\left(0 \cdots 0 \boldsymbol{k}_{\boldsymbol{i}}-10 \cdots 0 \boldsymbol{k}_{\jmath} 0 \cdots 0\right) \\
+\lambda_{0 \cdots 010 \cdots 010 \cdots 0} p\left(0 \cdots 0 \boldsymbol{k}_{\boldsymbol{i}}-10 \cdots 0 \boldsymbol{k}_{j}-10 \cdots 0\right) \\
\boldsymbol{k}_{\jmath} p\left(0 \cdots 0 \boldsymbol{k}_{\boldsymbol{\imath}} 0 \cdots 0 \boldsymbol{k}_{\jmath} 0 \cdots 0\right)=\lambda_{0 \cdots 000 \cdots 010 \cdots 0} p\left(0 \cdots 0 \boldsymbol{k}_{\boldsymbol{\imath}} 0 \cdots 0 \boldsymbol{k}_{j}-10 \cdots 0\right) \\
+\lambda_{0 \cdots 010 \cdots 010 \cdots 0} p\left(0 \cdots 0 \boldsymbol{k}_{\boldsymbol{i}}-10 \cdots 0 \boldsymbol{k}_{j}-10 \cdots 0\right)
\end{gathered}
$$

The selection of $\boldsymbol{i}$ and $j$ may be $\left(\begin{array}{l}n \\ 2\end{array}\right)$ cases, each case has two relations then we have totally $2\left(\begin{array}{l}n \\ 2\end{array}\right)$ relations and we need one of the relation in each case to do this so that we need $\left(\begin{array}{l}n \\ 2\end{array}\right)$ relations to do this. 
In the case of $\# \boldsymbol{k}=t(0<t \leqq n)$ if $\boldsymbol{k}_{\jmath_{1}}, \boldsymbol{k}_{\jmath_{2}}, \cdots, \boldsymbol{k}_{\jmath_{t}} \geqq 1$ for fixed $1 \leqq \jmath_{1}<\jmath_{2}<\cdots$ $<j_{t} \leqq n$, we have $t$ different relations and we need one of the relations to calculate the density. The selection of $j_{1}<\jmath_{2}<\cdots<\jmath_{t}$ may be in $\left(\begin{array}{l}n \\ t\end{array}\right)$ cases, so we have totally $t\left(\begin{array}{l}n \\ t\end{array}\right)$ relations and we need $\left(\begin{array}{l}n \\ t\end{array}\right)$ relations to do this. Additionary we can confirm each of the $t$ relations has $2^{t-1}$ terms in maximum in the right side of the relation; if $\lambda_{\boldsymbol{\imath}}>0$ for every $\boldsymbol{i} ; \boldsymbol{i} \in E, \boldsymbol{i} \leqq \boldsymbol{k}$ then our relation

$$
\boldsymbol{k}, p(\boldsymbol{k})=\sum_{\boldsymbol{i} \in E, \boldsymbol{\imath} \leqq k} \lambda_{\imath} p(\boldsymbol{k}-\boldsymbol{i})
$$

has maximum number of terms for $\boldsymbol{k}_{j} \geqq 1$. See DISCUSSION.

In the case of $\# \boldsymbol{k}=n, \boldsymbol{k}=\left(\boldsymbol{k}_{1}, \boldsymbol{k}_{2}, \cdots, \boldsymbol{k}_{n}\right)$ if any component of $\boldsymbol{k}$ is a positive integer then we have $n$ different relations and we need one of the $n$ relations to calculate the density. The selection of such a case may be $\left(\begin{array}{l}n \\ n\end{array}\right)$ case, so we have totally $n$ relations and we need one of the relations to calculate the density.

Proof of the theorem. Put $\# \boldsymbol{k}=1$ and $\boldsymbol{k}=\left(0 \cdots 0 \boldsymbol{k}_{j}, \cdots 0\right)$ where $\boldsymbol{k}_{\jmath}>0$ and assume $\boldsymbol{i} \leqq \boldsymbol{k}$ then $\boldsymbol{i}=(0 \cdots 010 \cdots 0)$. In the case we can use specified recurrence relation

then we have

$$
\boldsymbol{k}, p(\boldsymbol{k})=\lambda_{\imath} p(k-\boldsymbol{i}), \quad \boldsymbol{i}=\left(\begin{array}{llll}
0 & \cdots & 010 & \cdots
\end{array}\right)
$$

$$
p(\boldsymbol{k})=\left(\lambda_{\imath} / \boldsymbol{k}_{j}\right) p(\boldsymbol{k}-\boldsymbol{i}) .
$$

If $\boldsymbol{k}-\boldsymbol{i}=\left(0 \cdots 0 \boldsymbol{k}_{j}-10 \cdots 0\right) \neq 0$ in the right side, then we use the relation in iteration,

$$
\begin{aligned}
\boldsymbol{k}_{j}\left(\boldsymbol{k}_{j}-1\right) p(\boldsymbol{k}) & =\lambda_{i}\left(\boldsymbol{k}_{\jmath}-1\right) p(\boldsymbol{k}-\boldsymbol{i}) \\
& =\lambda_{i} 2 p(\boldsymbol{k}-2 \boldsymbol{i}) .
\end{aligned}
$$

By the iteration and $\boldsymbol{k}-\boldsymbol{k}_{j} \boldsymbol{i}=0$, we conclude

$$
\boldsymbol{k}_{\jmath} ! p(\boldsymbol{k})=\lambda_{i} \boldsymbol{k}_{j} p(0) .
$$

We can calculate all the density $p(\boldsymbol{k})$ for every $\boldsymbol{k}$ satisfying $\# \boldsymbol{k}=1$ and $\boldsymbol{k}_{j} \geqq 1$ for fiexed $j$ from $j$-th $r . r$. of type(1). From the arbitrariness of $\jmath$, we can calculate all the density $p(\boldsymbol{k})$ for every $\boldsymbol{k}$ satisfying $\# \boldsymbol{k} \leqq 1$, from our $n$ recurrence relations of type(1) $(j=1,2, \cdots, n)$. If we put $\# k=t$ then in the case $t=0(\boldsymbol{k}=0)$ we know that $p(\boldsymbol{k})$ is calculable from the relation. In the case $t=1$ $(\# \boldsymbol{k}=1)$ all the density $p(\boldsymbol{k})$ for $\# \boldsymbol{k}=1$ are calculable which is proved by using the recurrence relations of type(1) and the fact of $p(\boldsymbol{k})$ is calculable on $\boldsymbol{k}=0$. That is, the fact means all the density $p(\boldsymbol{k})$ are calculable on $\# \boldsymbol{k}$.

To proceed, in the proof by the induction for $t$. If $t=0$ then $p(\boldsymbol{k})$ is cal 
culable, and if $t=1$ then $p(\boldsymbol{k})$ is calculable. The proof is made using the fact $p(0)(t=0)$ is calculable. Therefore if we assume $p(\boldsymbol{k})$ is calculable for every $\boldsymbol{k}$ such that $\# \boldsymbol{k}<t$ and let prove that $p(\boldsymbol{k})$ is calculable on $\# \boldsymbol{k}=t$ using prescribed type $t$ recurrence relations. In the case $\# k=t$, we use our notations $\boldsymbol{i} \in E, E=\{0,1\}^{n}-0$ and $(\boldsymbol{k})_{\jmath}=\boldsymbol{k}_{\jmath}$. For every $\boldsymbol{k}_{\jmath}>0(1 \leqq j \leqq n)$ we can express the recurrence relation of type $t$

$$
\boldsymbol{k}_{\jmath} p(\boldsymbol{k})=\sum_{\left\{i ; \boldsymbol{i}_{j}=1, i \leq k\right\}} \lambda_{\imath} p(\boldsymbol{k}-\boldsymbol{i})
$$

where \{\} means $\left\{i ; \boldsymbol{i}_{\jmath}=1, \boldsymbol{i} \leqq \boldsymbol{k}, \boldsymbol{i} \in E\right\}=\left\{\boldsymbol{i} ;(\boldsymbol{i})_{\jmath}=1,0 \leqq \boldsymbol{k}-\boldsymbol{i}, \boldsymbol{i} \in E\right\}$, and put $\boldsymbol{i}_{1}$ the number of \{\} then we have $\#\left(\boldsymbol{k}-\boldsymbol{i}_{1}\right) \leqq t$. It $\#\left(\boldsymbol{k}-\boldsymbol{i}_{1}\right)<t$ then $p\left(\boldsymbol{k}-\boldsymbol{i}_{1}\right)$ in the right side of the relation $(*)$ is calculable from the assumption of the induction and otherwise if $\#\left(\boldsymbol{k}-\boldsymbol{i}_{1}\right)=t$ then $\left(\boldsymbol{k}-\boldsymbol{i}_{1}\right)_{j}=\boldsymbol{k}_{j}-1 \geqq 1$ and we can use the relation (*) of type $t$ for $p\left(\boldsymbol{k}-\boldsymbol{i}_{1}\right)$ one more time then we have

$$
\left(k-i_{1}\right)_{\jmath} p\left(k-i_{1}\right)=\sum_{\left(i ; i_{j}=1,1 \leqq k-i_{1}, i \in E\right)} \lambda_{\imath} p\left(k-i_{1}-i\right) .
$$

Put $\boldsymbol{i}_{2}$ the member of \{\} then we have $\#\left(\boldsymbol{k}-\boldsymbol{i}_{1}-\boldsymbol{i}_{2}\right) \leqq \#\left(\boldsymbol{k}-\boldsymbol{i}_{1}\right) \leqq t$. If $\#\left(\boldsymbol{k}-\boldsymbol{i}_{1}-\boldsymbol{i}_{2}\right)<t$ then $p\left(\boldsymbol{k}-\boldsymbol{i}_{1}-\boldsymbol{i}_{2}\right)$ in the right side of the relation is calculable from the assumption of the induction and otherwise if $\#\left(\boldsymbol{k}-\boldsymbol{i}_{1}-\boldsymbol{i}_{2}\right)=t$ then $\left(\boldsymbol{k}-\boldsymbol{i}_{1}-\boldsymbol{i}_{2}\right)_{\text {, }}$ $=\boldsymbol{k}_{j}-2 \geqq 1$ and we can use the relation $(*)$ of type $t$ for $p\left(\boldsymbol{k}-\boldsymbol{i}_{1}-\boldsymbol{i}_{2}\right)$ one more time then we have

$$
\left(k-i_{1}-i_{2}\right)_{j} p\left(k-i_{1}-i_{2}\right)=\sum_{\left(i ; i_{j}=1, \imath \leq k-i_{1}-i_{2}, i \in E\right)} \lambda_{\imath} p\left(k-i_{1}-i_{2}\right) .
$$

Put $\boldsymbol{i}_{3}$ the member of \{\} then we have $\#\left(\boldsymbol{k}-\boldsymbol{i}_{1}-\boldsymbol{i}_{2}-\boldsymbol{i}_{3}\right) \leqq t$. If $\#\left(\boldsymbol{k}-\boldsymbol{i}_{1}-\boldsymbol{i}_{2}-\boldsymbol{i}_{3}\right)$ $<t$ then $p\left(\boldsymbol{k}-\boldsymbol{i}_{1}-\boldsymbol{i}_{2}-\boldsymbol{i}_{3}\right)$ in the right side of the relation is calculate from the assumption (of the induction) and otherwise if $\#\left(k-\boldsymbol{i}_{1}-\boldsymbol{i}_{2}-\boldsymbol{i}_{3}\right)=t$ we have to continue this process so as to satisfy all the terms of the right side of the relation $(*)$ to be calculable. The process to get calculability require at most $\boldsymbol{k}_{j}-1$ steps and we put $s=\boldsymbol{k}_{j}-1$ then we have

$$
\left(\boldsymbol{k}_{j}-s\right) p\left(\boldsymbol{k}-\boldsymbol{i}_{1}-\boldsymbol{i}_{2}-\cdots-\boldsymbol{i}_{s}\right)=\sum_{\left\{\imath ; \boldsymbol{i}_{j}=1, \imath \leqq k-i_{1}-\boldsymbol{i}_{2}-\cdots-\boldsymbol{t}_{s}, \boldsymbol{i} \in E\right\}} \lambda_{\imath} p\left(\boldsymbol{k}-\boldsymbol{i}_{1}-\boldsymbol{i}_{2}-\cdots-\boldsymbol{i}_{s}-\boldsymbol{i}\right) .
$$

where $\#\left(\boldsymbol{k}-\boldsymbol{i}_{1}-\boldsymbol{i}_{2}-\cdots-\boldsymbol{i}_{s}\right) \leqq t$ and if $\#\left(\boldsymbol{k}-\boldsymbol{i}_{1}-\boldsymbol{i}_{2}-\cdots-\boldsymbol{i}_{s}\right)<t$ then the left side is already calculable by the assumption and otherwise, in the right side, $\#\left(k-\boldsymbol{i}_{1}-\boldsymbol{i}_{2}-\cdots-\boldsymbol{i}_{s}-\boldsymbol{i}\right)<t$ because $\left(\boldsymbol{k}-\boldsymbol{i}_{1}-\boldsymbol{i}_{2}-\cdots-\boldsymbol{i}_{s}-\boldsymbol{i}\right)_{j}=\boldsymbol{k}_{j}(s+1)=0$ for every member of \{\} . Therefore we have a result that all the terms of the right side of the relation $(*)$ are calculable where we used only the relation $(*)$ of type $t$ repeatedly and the assumption for the induction.

We recognize that the proof is made only on using the relation of type $t$ and disregard the other $t-1$ relations in the case $\# k=t$. And arbitrariness of $t$ (on $0 \leqq t \leqq n)$, we have the conclusion of the theorem.

Theorem 5-1. The density of $n$-variate Poisson distribution $p(\boldsymbol{k})$ for $\boldsymbol{k} \geqq 0$ is calculable from precribed $2^{n}$ relations including the relation 


$$
p(0)=\exp \left(-\sum_{i \in E} \lambda_{\boldsymbol{l}}\right) .
$$

If we omit the relation $p(0)=\exp \left(-\sum_{i \in E} \lambda_{\boldsymbol{l}}\right)$ and put $p(0)=p_{0}$ and using the other prescribed $2^{n-1}$ relations, we can calculate all the values $p(\boldsymbol{k})$ for $\boldsymbol{k} \geqq 0$ as the function of $p_{0}$ and if we introduce the assumption of the function to be a density, that is, summing up to unity then all the function $p(\boldsymbol{k}) \boldsymbol{k} \geqq 0$ consists with the multivariate Poisson density.

THEOREM 5-2. The function $p(\boldsymbol{k})$ for $\boldsymbol{k} \geqq 0$ is calculable from prescribed $2^{n}-1$ relations if we assume $p(\boldsymbol{k})$ for $\boldsymbol{k} \geqq 0$ to be a density.

The proof may be completed by putting $p(0)=p_{0}$ and using prescribed $2^{n}-1$ relations all the density is calculable as the multiple of $p_{0}$ in the same discussion of theorem 5-1 and $\sum_{k \geq 0} p(\boldsymbol{k})=1$. That is, the function is proved as $p_{0}$ calculable.

\section{DISCUSSION}

Through this paper, we have counted the number of relations and the number of terms in a relation including $\lambda_{l}=0$ for some of $i \in E$.

If we consider multivariate Poisson distribution usually we need to treat $\lambda_{\imath} \geqq 0$ for $i \in E$ then occasionally some parameters $\lambda_{\iota_{1}}, \cdots, \lambda_{\boldsymbol{t}_{t}}\left(\boldsymbol{i}_{1}, \cdots, \boldsymbol{i}_{t} \in E\right)$ are equal to zero.

So we have to understand the numbers in these theorems to be counted at most!

\section{REFERENCES}

[1] Johnson, N.L. and Kotz S., Discrete distributions, Houghton Millin Co. (1969)

[2] Kawamura, K., The structure of bivariate Poisson distribution, Kodai Math. Sem. Rep., $25(2)$ (1973), 246-256.

[3] Kawamura, K., The structure of trivariate Poisson distribution, Kodai Math Sem. Rep., 28 (1) (1976), 1-88.

[4] Kawamura, K., The structure of multivariate Poisson distribution, Kodai Math. J. 2 (3) (1979), 337-345.

[5] Kawamura, K., A note on the recurrence relations for the bivariate Poisson distribution, Kodai Math. J. 8(1) (1985), 70-78.

[6] LiU, C., Necessary and sufficient conditions for a Poisson approximation (trivariate case). Kodai Math. J. Vol. 9(3) (1986), 368-384.

[7] Polak, M., Poisson approximation for sums of independent bivariate Bernoulli vectors, Kodai Math. J. 8 (1982), 408-415. 